\title{
Classification of Cubic Symmetric Tricirculants
}

\author{
István Kovács ${ }^{a, 1}$ Klavdija Kutnar ${ }^{a, 1,2}$ Dragan Marušiča ${ }^{a, b, 1, *}$ Steve Wilson ${ }^{c}$ \\ ${ }^{a}$ University of Primorska, FAMNIT, Glagoljaška 8, 6000 Koper, Slovenia \\ ${ }^{b}$ University of Ljubljana, PEF, Kardeljeva pl. 16, 1000 Ljubljana, Slovenia \\ ${ }^{c}$ Northern Arizona University, Flagstaff, AZ 86011, USA
}

Submitted: Dec 16, 2011; Accepted: May 21, 2012; Published: May 31, 2012

\begin{abstract}
A tricirculant is a graph admitting a non-identity automorphism having three cycles of equal length in its cycle decomposition. A graph is said to be symmetric if its automorphism group acts transitively on the set of its arcs. In this paper it is shown that the complete bipartite graph $K_{3,3}$, the Pappus graph, Tutte's 8-cage and the unique cubic symmetric graph of order 54 are the only connected cubic symmetric tricirculants.
\end{abstract}

Keywords: symmetric graph, semiregular, tricirculant.

\section{Introductory remarks}

A graph is said to be arc-transitive, or symmetric, the term that will be used in this paper, if its automorphism group acts transitively on the set of arcs of the graph. A Cayley graph on a cyclic group, that is, a graph admitting an automorphism with a single cycle in its cycle decomposition, is said to be a circulant. A graph admitting a nonidentity automorphism with two (respectively, three) cycles of equal length in its cycle decomposition is said to be a bicirculant (respectively, tricirculant).

It is known that the complete graph $K_{4}$, the complete bipartite graph $K_{3,3}$ and the cube $Q_{3}$ are the only connected cubic symmetric graphs with girth (the length of shortest cycle) less than 5 (see [8, Proposition 3.4.]). Since any cubic circulant, being a Cayley

\footnotetext{
${ }^{1}$ Supported in part by "Agencija za raziskovalno dejavnost Republike Slovenije", research program P1-0285.

${ }^{2}$ The author gratefully acknowledges support by the US Department of State and the Fulbright Scholar Program, and thanks the Ohio State University for hospitality during her visit in Autumn 2010.

*Corresponding author e-mail: dragan.marusic@upr.si.
} 
graph on an abelian group, has girth less than or equal to 4 , one can easily see that $K_{4}$ and $K_{3,3}$ are the only examples of connected cubic symmetric circulants.

We may think of the classical result of Frucht, Graver and Watkins [7] in which they have classified all symmetric generalized Petersen graphs as the main step in the classification of all cubic connected symmetric bicirculants. The remaining cases were then completed in [19] and [21]. In particular, a connected cubic symmetric graph is a bicirculant if and only if it is isomorphic to one of the following graphs: the complete graph $K_{4}$, the complete bipartite graph $K_{3,3}$, the seven symmetric generalized Petersen graphs $\operatorname{GP}(4,1), \operatorname{GP}(5,2), \operatorname{GP}(8,3), \operatorname{GP}(10,2), \operatorname{GP}(10,3), \operatorname{GP}(12,5)$, and $\operatorname{GP}(24,5)($ see $[7,20])$, the Heawood graph F014A, and a Cayley graph Cay $\left(D_{2 n},\left\{b, b a, b a^{r+1}\right\}\right)$ on a dihedral group $D_{2 n}=\left\langle a, b \mid a^{n}=b^{2}=b a b a=1\right\rangle$ of order $2 n$ with respect to the generating set $\left\{b, b a, b a^{r+1}\right\}$, where $n \geqslant 11$ is odd and $r \in \mathbb{Z}_{n}^{*}$ such that $r^{2}+r+1 \equiv 0(\bmod n)$. (Hereafter the notation $\mathrm{F} n \mathrm{~A}, \mathrm{~F} n \mathrm{~B}$, etc. will refer to the corresponding graphs in the Foster census [3, 4].)

The aim of this paper is to move from bicirculants to tricirculants. A complete classification of connected cubic symmetric tricirculants is given. In particular, it is shown that the complete bipartite graph $K_{3,3}$ (which is also a circulant and a bicirculant), the Pappus graph F018A, Tutte's 8-cage F030A (sometimes also called the Tutte-Coxeter graph), and the graph F054A are the only connected cubic symmetric tricirculants, see Theorem 1.1. (For brevity in this paper, we will refer to these graphs as $K_{3,3}, \mathrm{~F} 018 \mathrm{~A}$, F030A and F054A, respectively.)

Theorem 1.1 A connected cubic symmetric graph $X$ is a tricirculant if and only if it is isomorphic to one of the following four graphs: $K_{3,3}, \mathrm{~F} 018 \mathrm{~A}, \mathrm{~F} 030 \mathrm{~A}$, and $\mathrm{F} 054 \mathrm{~A}$.

The classification is obtained by first considering the so-called core-free tricirculants, that is, tricirculants admitting a non-identity automorphism $\rho$, having three cycles of equal length in its cycle decomposition, such that the subgroup generated by $\rho$ is core-free in the full automorphism group of the graph. A remarkable group-theoretic result of Herzog and Kaplan [11], which says that 'sufficiently large' cyclic subgroups are never corefree (see Lemma 3.2), combined together with the well-known fact that the automorphism group of a connected cubic symmetric graph of order $n$ is of order $3 \cdot 2^{s-1} n$, where $s \leqslant 5$, enable us to prove that $K_{3,3}, \mathrm{~F} 018 \mathrm{~A}$, and F030A are the only connected cubic symmetric core-free tricirculants (see Theorem 3.3). Whereas, for non-core-free cubic symmetric tricirculants Lorimer's result about cubic symmetric graphs admitting a normal subgroup in its automorphism group implies that any such graph is a regular cyclic cover of a cubic symmetric core-free tricirculant (see Lemma 3.4). This then enables us to use graph covering techniques, which we recall in Subsection 2.2, to prove that the graph F054A is the only connected cubic symmetric non-core-free tricirculant, and the classification follows.

\section{Preliminaries}

Throughout this paper graphs are simple, finite, undirected and connected. Given a 
graph $X$ we let $V(X), E(X), A(X)$ and Aut $X$ be the vertex set, the edge set, the arc set and the automorphism group of $X$, respectively. A sequence of $k+1$ vertices in $X$, not necessarily all distinct, such that any two consecutive vertices are adjacent and any three consecutive vertices are distinct is called a $k$-arc.

A subgroup $G \leqslant$ Aut $X$ is said to be vertex-transitive, edge-transitive and arc-transitive provided it acts transitively on the sets of vertices, edges and $\operatorname{arcs}$ of $X$, respectively. The graph $X$ is said to be vertex-transitive, edge-transitive, and arc-transitive if its automorphism group is vertex-transitive, edge-transitive and arc-transitive, respectively. An arc-transitive graph is also called symmetric. A subgroup $G \leqslant$ Aut $X$ is said to be $s$ arc-transitive if it acts transitively on the set of $s$-arcs, and it is said to be $s$-regular if it is $s$-arc-transitive and the stabilizer of an $s$-arc in $G$ is trivial. A graph is said to be $s$-regular if its automorphism group is s-regular. By Tutte's result [22] every connected cubic symmetric graph is $s$-regular for some $s \leqslant 5$.

For a partition $\mathcal{W}$ of $V(X)$, we let $X_{\mathcal{W}}$ be the associated quotient graph of $X$ relative to $\mathcal{W}$, that is, the graph with vertex set $\mathcal{W}$ and edge set induced naturally by the edge set $E(X)$. In the case when $\mathcal{W}$ corresponds to the set of orbits of a subgroup $N$ of Aut $X$, the symbol $X_{\mathcal{W}}$ will be replaced by $X_{N}$.

\subsection{Semiregular automorphisms}

A non-identity automorphism of a graph is semiregular, in particular, $(k, n)$-semiregular if it has $k$ cycles of equal length $n$ in its cycle decomposition. An $n$-tricirculant (tricirculant for short) is a graph with a $(3, n)$-semiregular automorphism.

Let $X$ be a connected graph admitting a $(k, n)$-semiregular automorphism

$$
\rho=\left(u_{0}^{0} u_{0}^{1} \cdots u_{0}^{n-1}\right)\left(u_{1}^{0} u_{1}^{1} \cdots u_{1}^{n-1}\right) \cdots\left(u_{k-1}^{0} u_{k-1}^{1} \cdots u_{k-1}^{n-1}\right),
$$

and let $\mathcal{W}=\left\{W_{i} \mid i \in \mathbb{Z}_{k}\right\}$ be the set of orbits $W_{i}=\left\{u_{i}^{s} \mid s \in \mathbb{Z}_{n}\right\}$ of $\rho$. Using Frucht's notation [6] $X$ may be represented in the following way. Each orbit of $\rho$ is represented by a circle. Inside a circle corresponding to the orbit $W_{i}$ the symbol $n / T$, where $T=T^{-1} \subseteq \mathbb{Z}_{n} \backslash\{0\}$, indicates that for each $s \in \mathbb{Z}_{n}$, the vertex $u_{i}^{s}$ is adjacent to all the vertices $u_{i}^{s+t}$ where $t \in T$. When $|T| \leqslant 2$ we use a simplified notation $n / t, n /(n / 2)$ and $n$, when, respectively, $T=\{t,-t\}, T=\{n / 2\}$ and $T=\emptyset$. Finally, an arrow pointing from the circle representing the orbit $W_{i}$ to the circle representing the orbit $W_{j}, j \neq i$, labeled by $y \in \mathbb{Z}_{n}$ means that for each $s \in \mathbb{Z}_{n}$, the vertex $u_{i}^{s} \in W_{i}$ is adjacent to the vertex

$u_{j}^{s+y}$. When the label is 0, the arrow on the line may be omitted. Examples illustrating this notation are given in Figure 1.

\subsection{Graph Covers}

A covering projection of a graph $\widetilde{X}$ is a surjective mapping $p: \widetilde{X} \rightarrow X$ such that for each $\tilde{u} \in V(\tilde{X})$ the set of arcs emanating from $\tilde{u}$ is mapped bijectively onto the set of arcs emanating from $u=p(\tilde{u})$. The graph $\widetilde{X}$ is called a covering graph of the base graph $X$. The set $\mathrm{fib}_{u}=p^{-1}(u)$ is a fibre of a vertex $u \in V(X)$. The subgroup $K$ of 

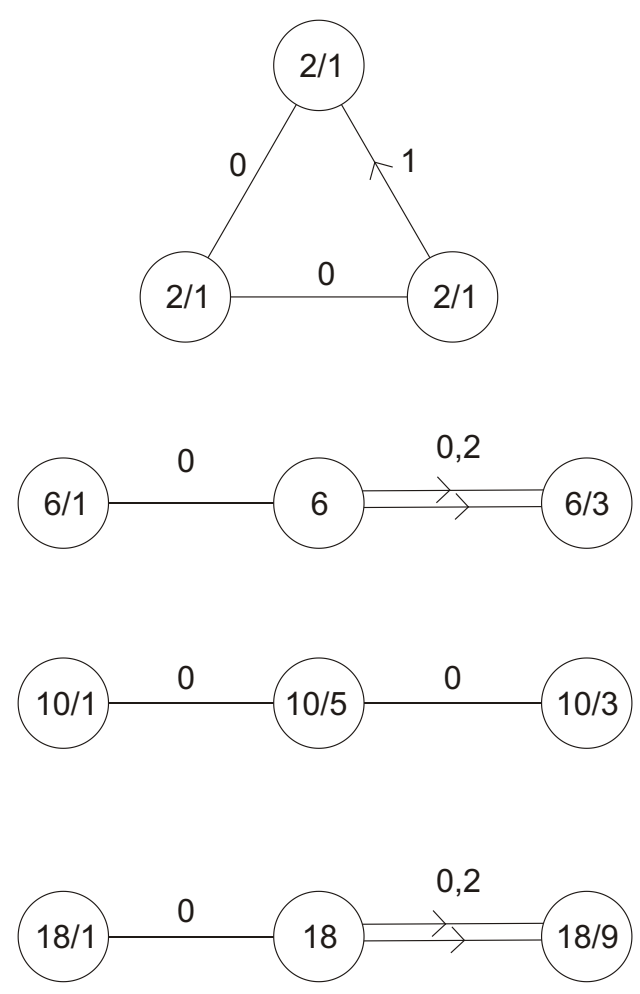

Figure 1: The graphs $K_{3,3}$, F018A, F030A and F054A shown in Frucht's notation with respect to a $(3,2)-,(3,6)-,(3,10)$ - and $(3,18)$-semiregular automorphism, respectively.

all those automorphisms of $\tilde{X}$ which fix each of the fibres setwise is called the group of covering transformations. The graph $\tilde{X}$ is also called a $K$-cover of $X$. It is a simple observation that the group of covering transformations of a connected covering graph acts semiregularly on each of the fibres. In particular, if the group of covering transformations is regular on the fibres of $\tilde{X}$, we say that $\tilde{X}$ is a regular $K$-cover. We say that $\alpha \in \operatorname{Aut} X$ lifts to an automorphism of $\tilde{X}$ if there exists an automorphism $\tilde{\alpha} \in$ Aut $\tilde{X}$, called a lift of $\alpha$, such that $\tilde{\alpha} p=p \alpha$. If the covering graph $\widetilde{X}$ is connected then $K$ is the lift of the trivial subgroup of Aut $X$. Note that a subgroup $G \leqslant \operatorname{Aut} \widetilde{X}$ projects if and only if the partition of $V(\tilde{X})$ into the orbits of $K$ is $G$-invariant.

A combinatorial description of a $K$-cover was introduced through a voltage graph by Gross and Tucker [10] as follows. Let $X$ be a graph and $K$ be a finite group. A voltage assignment of $X$ is a mapping $\zeta: A(X) \rightarrow K$ with the property that $\zeta(u, v)=\zeta(v, u)^{-1}$ for any arc $(u, v) \in A(X)$ (here, and in the rest of the paper, $\zeta(u, v)$ is written instead of $\zeta((u, v))$ for the sake of brevity). The voltage assignment $\zeta$ extends to walks in $X$ in a natural way. In particular, for any walk $D=u_{1} u_{2} \cdots u_{t}$ of $X$ we let $\zeta(D)$ denote the product voltage $\zeta\left(u_{1}, u_{2}\right) \zeta\left(u_{2}, u_{3}\right) \cdots \zeta\left(u_{t-1}, u_{t}\right)$ of $D$, that is, the $\zeta$-voltage of $D$. 
The values of $\zeta$ are called voltages, and $K$ is the voltage group. The voltage graph $X \times_{\zeta} K$ derived from a voltage assignment $\zeta: A(X) \rightarrow K$ has vertex set $V(X) \times K$, and edges of the form $\{(u, g),(v, \zeta(x) g)\}$, where $x=(u, v) \in A(X)$. Clearly, $X \times_{\zeta} K$ is a covering of $X$ with the first coordinate projection. By letting $K$ act on $V\left(X \times_{\zeta} K\right)$ as $(u, g)^{g^{\prime}}=\left(u, g g^{\prime}\right),(u, g) \in V\left(X \times_{\zeta} K\right), g^{\prime} \in K$, one obtains a semiregular group of automorphisms of $X \times_{\zeta} K$, showing that $X \times_{\zeta} K$ can in fact be viewed as a $K$-cover of $X$.

Given a spanning tree $T$ of $X$, the voltage assignment $\zeta: A(X) \rightarrow K$ is said to be $T$-reduced if the voltages on the tree arcs equal the identity element in $K$. In [9] it is shown that every regular covering graph $\tilde{X}$ of a graph $X$ can be derived from a $T$-reduced voltage assignment $\zeta$ with respect to an arbitrary fixed spanning tree $T$ of $X$.

The problem of whether an automorphism $\alpha$ of $X$ lifts or not is expressed in terms of voltages as follows (see Proposition 2.1). Given $\alpha \in$ Aut $X$ and the set of fundamental closed walks $\mathcal{C}$ based at a fixed vertex $v \in V(X)$, we define $\bar{\alpha}=\left\{\left(\zeta(C), \zeta\left(C^{\alpha}\right)\right) \mid C \in\right.$ $\mathcal{C}\} \subseteq K \times K$. Note that if $K$ is abelian, $\bar{\alpha}$ does not depend on the choice of the base vertex, and the fundamental closed walks at $v$ can be substituted by the fundamental cycles generated by the cotree arcs of $X$. Also, from the definition, it is clear that for a $T$-reduced voltage assignment $\zeta$ the derived graph $X \times_{\zeta} K$ is connected if and only if the voltages of the cotree arcs generate the voltage group $K$.

We wrap up this section with four propositions dealing with lifting of automorphisms in graph covers. The first one may be deduced from [17, Theorem 4.2], the second one from [12] whereas the third one is taken from [5, Proposition 2.2], but it may also be deduced from [18, Corollaries 9.4, 9.7, 9.8].

Proposition 2.1 [17] Let $K$ be a finite group, and let $X \times{ }_{\zeta} K$ be a connected regular cover of a graph $X$ derived from a voltage assignment $\zeta$ with the voltage group $K$. Then an automorphism $\alpha$ of $X$ lifts if and only if $\bar{\alpha}$ is a function which extends to an automorphism $\alpha^{*}$ of $K$.

For a connected regular cover $X \times{ }_{\zeta} K$ of a graph $X$ derived from a $T$-reduced voltage assignment $\zeta$ with an abelian voltage group $K$ and an automorphism $\alpha \in \operatorname{Aut} X$ that lifts, $\bar{\alpha}$ will always denote the mapping from the set of voltages of the fundamental cycles on $X$ to the voltage group $K$ and $\alpha^{*}$ will denote the automorphism of $K$ arising from $\bar{\alpha}$.

Two coverings $p_{i}: \widetilde{X}_{i} \rightarrow X, i \in\{1,2\}$, are said to be isomorphic if there exists a graph isomorphism $\phi: \widetilde{X}_{1} \rightarrow \widetilde{X}_{2}$ such that $\phi p_{2}=p_{1}$.

Proposition 2.2 [12] Let $K$ be a finite group. Two connected regular covers $X \times_{\zeta} K$ and $X \times_{\varphi} K$, where $\zeta$ and $\varphi$ are T-reduced, are isomorphic if and only if there exists an automorphism $\sigma \in$ AutK such that $\zeta(u, v)^{\sigma}=\varphi(u, v)$ for any cotree arc $(u, v)$ of $X$.

Proposition 2.3 [5] Let $K$ be a finite group, and let $X \times_{\zeta} K$ be a connected regular cover of a graph $X$ derived from a voltage assignment $\zeta$ with the voltage group $K$, and let the lifts of $\alpha \in A u t X$ centralize $K$, considered as the group of covering transformations. Then for any closed walk $W$ in $X$, there exists $k \in K$ such that $\zeta\left(W^{\alpha}\right)=k \zeta(W) k^{-1}$. In particular, if $K$ is abelian, $\zeta\left(W^{\alpha}\right)=\zeta(W)$ for any closed walk $W$ of $X$. 
Given a voltage assignment $\zeta$ of $X$ and $\beta \in$ Aut $X$, we set $\zeta^{\beta}$ for the voltage assignment of $X$ such that $\zeta^{\beta}(u, v)=\zeta\left(u^{\beta^{-1}}, v^{\beta^{-1}}\right),(u, v) \in A(X)$; and set $\widetilde{\beta}$ for the permutation of $V(X) \times K$ acting as $(u, k)^{\widetilde{\beta}}=\left(u^{\beta}, k\right)$. Our last proposition is straightforward.

Proposition 2.4 Let $K$ be a finite group, and let $\widetilde{X}=X \times_{\zeta} K$ be a connected regular cover of a graph $X$ derived from a voltage assignment $\zeta$ with the voltage group $K$, and let $\beta \in$ Aut $X$. Then the following hold.

(i) $\widetilde{\beta}$ is an isomorphism from $\widetilde{X}$ to $X \times_{\zeta^{\beta}} K$.

(ii) If $\widetilde{\alpha}$ is in Aut $\widetilde{X}$ which projects to $\alpha$, then $\widetilde{\beta}^{-1} \widetilde{\alpha} \widetilde{\beta}$ is in $\operatorname{Aut}\left(X \times_{\zeta^{\beta}} K\right)$, and it projects to $\beta^{-1} \alpha \beta$.

(iii) If $\widetilde{\alpha} \in$ Aut $\widetilde{X}$ centralizes the group $K$ of covering transformations, then also $\widetilde{\beta}^{-1} \widetilde{\alpha} \widetilde{\beta}$ centralizes $K$.

\section{Cubic symmetric tricirculants}

Let $\mathcal{T C}$ be the family of connected cubic symmetric tricirculants. We are going to show that $K_{3,3}, \mathrm{~F} 018 \mathrm{~A}, \mathrm{~F} 030 \mathrm{~A}$ and F054A, the four graphs shown in Figure 1, are the only connected cubic symmetric tricirculants. The graph F018A is the unique connected cubic symmetric graph of order 18. It is 3-regular, has girth 6 and is the Levi graph of the Pappus configuration. The graph F030A is the unique connected cubic symmetric graph of order 30. It is 5-regular, has girth 8 and diameter 4 . As the unique smallest cubic graph of girth 8 it is a cage and a Moore graph (see also [13]). It is bipartite, and can be constructed as the Levi graph of the generalized quadrangle $G Q(2,2)$. The graph F054A is the unique cubic symmetric graph of order 54. It is 2-regular and has girth 6 . For more information on these graphs we refer the reader to $[3,4,14,16]$.

Using the table of cubic symmetric graphs of order up to 768 in $[3,4]$ and a program package MAGMA [2] one can see that the following lemma holds.

Lemma 3.1 There is no cubic 1-regular tricirculant of order less than 27. The graphs F024A, F048A, F090A, F096B, F102A, F204A, and F234B are not tricirculants.

Recall that the core of a subgroup $K$ in a group $G$ (denoted by core $_{G}(K)$ ) is the largest normal subgroup of $G$ contained in $K$. A graph $X \in \mathcal{T C}$ with a $(3, n)$-semiregular automorphism is said to be core-free if there exists a $(3, n)$-semiregular automorphism $\rho \in$ Aut $X$ such that the cyclic subgroup $\langle\rho\rangle$ has trivial core in Aut $X$. To obtain the classification of cubic symmetric core-free tricirculants (see Theorem 3.3) the following group-theoretical result will be used.

Lemma 3.2 [11, Theorem B] If $H$ is a cyclic subgroup of a finite group $G$ with $|H| \geqslant$ $\sqrt{|G|}$, then $H$ contains a non-trivial normal subgroup of $G$. 
Theorem 3.3 A graph $X \in \mathcal{T C}$ is core-free if and only if it is isomorphic to one of the following three graphs: $K_{3,3}, \mathrm{~F} 018 \mathrm{~A}$, and F030A.

Proof. Let $X$ be cubic $s$-arc-transitive tricirculant of order $o=3 n$, and let $G=$ Aut $X$. Then, by [1, Proposition 18.1]), $G$ is regular on the set of $s$-arcs of $X$. By Tutte's theorem (see [1, Theorem 18.6]) we have that $s \leqslant 5$, and therefore $|G|=9 \cdot 2^{s-1} \cdot n \leqslant 144 n$. Since $X$ is core-free, Lemma 3.2 implies that $n^{2}<|G|$, and consequently $n<144$.

In particular, if $s=1$ then $n^{2}<|G|=9 n$, implying that $n<9$ and $o=3 n<27$, which in view of Lemma 3.1 is impossible.

If $s=2$ then $n^{2}<|G|=18 n$, implying that $n<18$ and $o=3 n<54$. By the table of cubic symmetric graphs [4] the only cubic 2-regular graphs of order less than 54 (and divisible by 3) are F024A and F048A. However, by Lemma 3.1, F024A and F048A are not tricirculants.

If $s=3$ then $n^{2}<|G|=36 n$, and so $n<36$ and $o=3 n<108$. The only cubic 3 -regular graphs satisfying this condition are the graphs $K_{3,3}$, F018A and F096B. The first two graphs are clearly tricirculants whereas the latter graph is not a tricirculant by Lemma 3.1.

If $s=4$ then $n^{2}<|G|=72 n$, and so $n<72$ and $o=3 n<216$. The only cubic 4-regular graphs satisfying this condition are the graphs F102A and F204A, which, by Lemma 3.1, are not tricirculants.

If $s=5$ then $n^{2}<|G|=144 n$, and so $n<144$ and $o=3 n<432$. The only cubic 5-regular graphs satisfying this condition are the graphs F030A, F090A and F234B. However, the last two graphs, by Lemma 3.1, are not tricirculants.

That $K_{3,3}, \mathrm{~F} 018 \mathrm{~A}$, and F030A are indeed core-free can be easily checked with the use of MAGMA [2].

The following lemma follows from [15, Theorem 9].

Lemma 3.4 Let $X \in \mathcal{T C}$ with a $(3, n)$-semiregular automorphism $\rho \in$ Aut $X$, and let $N$ be the core of $\langle\rho\rangle$ in Aut $X$. Then $N$ is the kernel of AutX acting on the set of orbits of $N$, Aut $X / N$ acts arc-transitively on $X_{N}, X_{N} \in \mathcal{T C}$ with a $(3, n /|N|)$-semiregular automorphism and $X_{N}$ is core-free.

We are now ready to prove the main theorem of this paper.

Proof of Theorem 1.1. Let $X$ be a cubic symmetric tricirculant with a $(3, n)$ semiregular automorphism $\rho \in \operatorname{Aut} X$. If $X$ is core-free then, by Theorem $3.3, X$ is isomorphic to $K_{3,3}$, or F018A, or F030A.

Suppose now that $X$ is not core-free. Then there exists a nontrivial subgroup $N$ of $\langle\rho\rangle$ which is normal in Aut $X$. By Lemma 3.4, the quotient graph $X_{N}$ is a connected cubic symmetric core-free tricirculant, and hence, by Theorem 3.3, it is isomorphic to $K_{3,3}$, F018A or F030A. In fact, $X$ is isomorphic to a regular $\mathbb{Z}_{r}$-cover of one of these three graphs, where $|N|=r$. Note also that $\rho$ projects to a $(3, n / r)$-semiregular automorphism of $X_{N}$. (Below, all arithmetic operations are to be taken modulo $r$ if at least one argument is from $\mathbb{Z}_{r}$ and the symbol mod $r$ is always omitted.) 


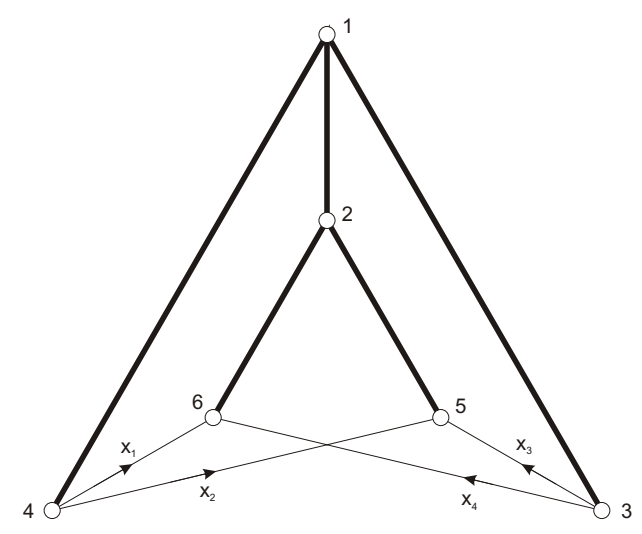

Figure 2: The voltage assignment $\zeta$ on $K_{3,3}$. The spanning tree consists of undirected bold edges, all carrying trivial voltage.

CASE $1 . X_{N} \cong K_{3,3}$.

The graph $K_{3,3}$ is illustrated in Figure 2. It is known that $K_{3,3}$ is the unique connected cubic symmetric graph of order 6 and that this graph is in fact 3-regular (see [4]). Let us choose the following automorphisms of $K_{3,3}$

$$
\alpha=(1)(2,4,3)(5)(6) \text { and } \beta=(1,2)(3,5)(4,6),
$$

and let $H=\langle\alpha, \beta\rangle$. It can be checked directly, using Magma [2], that every $(3,2)$ semiregular automorphism of $K_{3,3}$ is conjugate to $\beta$, and that every arc-transitive subgroup of Aut $K_{3,3}$, which contains $\beta$, must contain also the subgroup $H$. Because of Proposition 2.4 we may assume without loss of generality that $\rho$ projects to $\beta$ (therefore, the lifts of $\beta$ centralize the group $N$ of covering transformations) and that $H$ lifts to a subgroup of Aut $X$.

Any such cover $X$ can be derived from $K_{3,3}$ through a suitable voltage assignment $\zeta: A\left(K_{3,3}\right) \rightarrow \mathbb{Z}_{r}$. To find this voltage assignment $\zeta$ fix the spanning tree $T$ of $K_{3,3}$ as the one consisting of the edges

$$
\{1,2\},\{1,3\},\{1,4\},\{2,5\},\{2,6\}
$$

(see also Figure 2). There are four fundamental cycles in $K_{3,3}$, which are generated, respectively, by four cotree arcs $(4,6),(4,5),(3,5)$, and $(3,6)$. By Proposition $2.3, \beta^{*}$ is the identity automorphism of $\mathbb{Z}_{r}$, and thus we get from Table 1 , where all these cycles and their voltages are listed, that $2 x_{1}=2 x_{3}=0$ and $x_{2}=-x_{4}$. Moreover, since $\alpha$ lifts, Proposition 2.1 implies that $\bar{\alpha}$ is a function which extends to an automorphism $\alpha^{*}$ of $\mathbb{Z}_{r}$. Therefore, since $\zeta\left(C_{3}^{\alpha}\right)=-x_{2}$ and $\zeta\left(C_{4}^{\alpha}\right)=-x_{1}$, it follows that all non-trivial voltages are of order at most 2. Since $X$ is assumed to be connected at least one non-trivial voltage exists and the set of all non-trivial voltages generates the voltage group. Since the voltage group is cyclic, it follows that $r=2$. But, however, there is no connected cubic symmetric graph of order 12, and so this case is impossible. 


\begin{tabular}{c|c|c|c|c|c|c}
\hline & $C$ & $\zeta(C)$ & $C^{\alpha}$ & $\zeta\left(C^{\alpha}\right)$ & $C^{\beta}$ & $\zeta\left(C^{\beta}\right)$ \\
\hline \hline$C_{1}$ & $1,4,6,2,1$ & $x_{1}$ & $1,3,6,4,1$ & $x_{4}-x_{1}$ & $2,6,4,1,2$ & $-x_{1}$ \\
$C_{2}$ & $1,4,5,2,1$ & $x_{2}$ & $1,3,5,4,1$ & $x_{3}-x_{2}$ & $2,6,3,1,2$ & $-x_{4}$ \\
$C_{3}$ & $1,3,5,2,1$ & $x_{3}$ & $1,2,5,4,1$ & $-x_{2}$ & $2,5,3,1,2$ & $-x_{3}$ \\
$C_{4}$ & $1,3,6,2,1$ & $x_{4}$ & $1,2,6,4,1$ & $-x_{1}$ & $2,5,4,1,2$ & $-x_{2}$ \\
\hline \hline
\end{tabular}

Table 1: Fundamental cycles and their images with corresponding voltages in $K_{3,3}$.

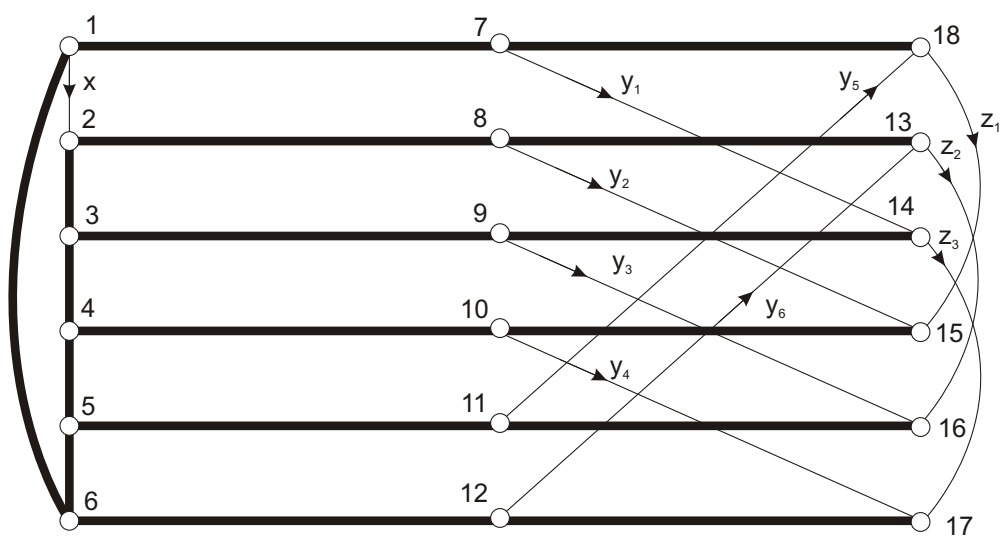

Figure 3: The voltage assignment $\zeta$ on F018A. The spanning tree consists of undirected bold edges, all carrying trivial voltage.

\section{CASE $2 . X_{N} \cong \mathrm{F} 018 \mathrm{~A}$.}

The graph F018A is illustrated in Figure 3. It is known that F018A is the unique connected cubic symmetric graph of order 18 and that this graph is in fact 3-regular (see [4]). Let us choose the following automorphisms of F018A

$$
\begin{aligned}
\alpha & =(1,2)(3,6)(4,5)(7,8)(9,12)(10,11)(13,14)(15,18)(16,17), \\
\beta & =(1,7,14,9,3,2)(4,8,6,18,17,16)(5,15,12,11,10,13), \\
\gamma_{1} & =(3,8)(4,15)(5,18)(6,7)(9,13)(12,14), \\
\gamma_{2} & =(2,6)(3,5)(8,12)(9,11)(14,18)(15,17), \\
\gamma_{3} & =(1,2)(3,6,8,7)(4,12,15,14)(5,13,18,9)(10,17)(11,16), \\
\delta & =(4,9)(5,14)(6,7)(10,16)(11,17)(12,18)(13,15) .
\end{aligned}
$$

Then AutF018A $=\left\langle\alpha, \beta, \gamma_{3}, \delta\right\rangle=\left\langle\gamma_{1}, \gamma_{2}, \gamma_{3}, \delta\right\rangle$. Each subgroup of AutF018A generated by a $(3,6)$-semiregular automorphism is conjugate to $\langle\beta\rangle$, and each proper arc-transitive subgroup of AutF018A is conjugate in AutF018A to one of the three subgroups $H_{1}=\langle\alpha, \beta\rangle$, $H_{2}=\left\langle\alpha, \beta, \gamma_{1}\right\rangle$ and $H_{3}=\left\langle\gamma_{1}, \gamma_{2}, \gamma_{3}\right\rangle$. In addition, $H_{1}$ is 1-regular, $H_{2}$ and $H_{3}$ are 2-regular, 
$\langle\beta\rangle \leqslant H_{1} \leqslant H_{2}$, and $H_{3}$ does not contain a $(3,6)$-semiregular automorphism. These can be checked directly using Magma [2]. Thus it suffices to find those arc-transitive regular $\mathbb{Z}_{r}$-covers of F018A for which the subgroup $H_{1}$ lifts and the lifts of the automorphism $\beta$ centralizing the group of covering transformations and having precisely 3 orbits of size $n$.

\begin{tabular}{c|c|c|c|c|c}
\hline Row & & $C$ & $\zeta(C)$ & $C^{\alpha}$ & $\zeta\left(C^{\alpha}\right)$ \\
\hline \hline A.1 & $C_{0}$ & $1,2,3,4,5,6,1$ & $x$ & $2,1,6,5,4,3,2$ & $-x$ \\
A.2 & $C_{1}$ & $1,7,14,9,3,4,5,6,1$ & $y_{1}$ & $2,8,13,12,6,5,4,3,2$ & $-y_{6}$ \\
A.3 & $C_{2}$ & $2,8,15,10,4,3,2$ & $y_{2}$ & $1,7,18,11,5,6,1$ & $-y_{5}$ \\
A.4 & $C_{3}$ & $3,9,16,11,5,4,3$ & $y_{3}$ & $6,12,17,10,4,5,6$ & $-y_{4}$ \\
A.5 & $C_{4}$ & $4,10,17,12,6,5,4$ & $y_{4}$ & $5,11,16,9,3,4,5$ & $-y_{3}$ \\
A.6 & $C_{5}$ & $5,11,18,7,1,6,5$ & $y_{5}$ & $4,10,15,8,2,3,4$ & $-y_{2}$ \\
A.7 & $C_{6}$ & $6,12,13,8,2,3,4,5,6$ & $y_{6}$ & $3,9,14,7,1,6,5,4,3$ & $-y_{1}$ \\
A.8 & $C_{7}$ & $1,7,18,15,10,4,5,6,1$ & $z_{1}$ & $2,8,15,18,11,5,4,3,2$ & $y_{2}-z_{1}-y_{5}$ \\
A.9 & $C_{8}$ & $2,8,13,16,11,5,4,3,2$ & $z_{2}$ & $1,7,14,17,10,4,5,6,1$ & $y_{1}+z_{3}-y_{4}$ \\
A.10 & $C_{9}$ & $3,9,14,17,12,6,5,4,3$ & $z_{3}$ & $6,12,13,16,9,3,4,5,6$ & $y_{6}+z_{2}-y_{3}$ \\
\hline \hline & & & $\zeta(C)$ & & $C^{\beta}$ \\
\hline \hline B.1 & $C_{0}$ & $1,2,3,4,5,6,1$ & $x$ & $7,1,2,8,15,18,7$ & $\zeta\left(C^{\beta}\right)$ \\
B.2 & $C_{1}$ & $1,7,14,9,3,4,5,6,1$ & $y_{1}$ & $7,14,9,3,2,8,15,18,7$ & $y_{1}+y_{2}-z_{1}$ \\
B.3 & $C_{2}$ & $2,8,15,10,4,3,2$ & $y_{2}$ & $1,6,12,13,8,2,1$ & $y_{6}-x$ \\
B.4 & $C_{3}$ & $3,9,16,11,5,4,3$ & $y_{3}$ & $2,3,4,10,15,8,2$ & $-y_{2}$ \\
B.5 & $C_{4}$ & $4,10,17,12,6,5,4$ & $y_{4}$ & $8,13,16,11,18,15,8$ & $z_{2}+y_{5}+z_{1}-y_{2}$ \\
B.6 & $C_{5}$ & $5,11,18,7,1,6,5$ & $y_{5}$ & $15,10,17,14,7,18,15$ & $y_{4}-z_{3}-y_{1}+z_{1}$ \\
B.7 & $C_{6}$ & $6,12,13,8,2,3,4,5,6$ & $y_{6}$ & $18,11,5,6,1,2,8,15,18$ & $-y_{5}+x+y_{2}-z_{1}$ \\
B.8 & $C_{7}$ & $1,7,18,15,10,4,5,6,1$ & $z_{1}$ & $7,14,17,12,13,8,15,18,7$ & $y_{1}+z_{3}+y_{6}+y_{2}-z_{1}$ \\
B.9 & $C_{8}$ & $2,8,13,16,11,5,4,3,2$ & $z_{2}$ & $1,6,5,4,10,15,8,2,1$ & $-y_{2}-x$ \\
B.10 & $C_{9}$ & $3,9,14,17,12,6,5,4,3$ & $z_{3}$ & $2,3,9,16,11,18,15,8,2$ & $y_{3}+y_{5}+z_{1}-y_{2}$ \\
\hline \hline
\end{tabular}

Table 2: Fundamental cycles and their images with corresponding voltages in F018A.

The graph $X$ can be derived from F018A through a suitable voltage assignment $\zeta: A(\mathrm{~F} 018 \mathrm{~A}) \rightarrow \mathbb{Z}_{r}$. To find this voltage assignment $\zeta$ fix the spanning tree $T$ of $\mathrm{F} 018 \mathrm{~A}$ as the one consisting of the edges

$$
\begin{gathered}
\{1,7\},\{2,8\},\{3,9\},\{4,10\},\{5,11\},\{6,12\},\{7,18\},\{8,13\}, \\
\{9,14\},\{10,15\},\{11,16\},\{12,17\},\{2,3\},\{3,4\},\{4,5\},\{5,6\},\{6,1\}
\end{gathered}
$$

(see also Figure 3). There are ten fundamental cycles in F018A, which are generated, respectively, by ten cotree arcs $(1,2),(7,14),(8,15),(9,16),(10,17),(11,18),(12,13)$, $(18,15),(13,16)$ and $(14,17)$ (see Table 2 where all these cycles and their voltages are listed).

Now let us consider the mappings $\bar{\alpha}$ and $\bar{\beta}$ from the set $S=\left\{x, y_{i}, z_{j} \mid i \in\{1,2, \ldots, 6\}\right.$, $j \in\{1,2,3\}\}$ of voltages of the ten fundamental cycles of F018A to the voltage group $\mathbb{Z}_{r}$. Since $X$ is connected we have $\mathbb{Z}_{r}=\langle S\rangle$. Proposition 2.1 implies that the mappings $\bar{\alpha}$ and $\bar{\beta}$ are extended to automorphisms $\alpha^{*}$ and $\beta^{*}$ of $\mathbb{Z}_{r}$, respectively. Also, since the lifts of $\beta$ centralize the group of covering transformations, Proposition 2.3 implies that $\beta^{*}$ is the identity automorphism of $\mathbb{Z}_{r}$. Therefore, it follows from Rows B.1 and B.4 of Table 2 that $y_{2}=z_{1}=-y_{3}$. By Rows A.3 and A.4 of Table 2, we get that $y_{2}^{\alpha^{*}}=-y_{5}$ and $y_{3}^{\alpha^{*}}=-y_{4}$, and so $y_{5}=-y_{4}$. In other words, $y_{2}, y_{3}, y_{4}, y_{5}$ and $z_{1}$ are of the same 
order and so $y_{2}, y_{3}, y_{4}, z_{1} \in\left\langle y_{5}\right\rangle$. Further, by Rows B.5 and B.7 of Table 2 we get that $z_{2}=2 y_{4}=-2 y_{5} \in\left\langle y_{5}\right\rangle$ and $x=y_{5}+y_{6}$, respectively, implying that in fact $\mathbb{Z}_{r}=\left\langle y_{i}, z_{3}\right|$ $i \in\{1,5,6\}\rangle$. In addition, by Row B.10 of Table 2, we have $z_{3}=y_{3}+y_{5} \in\left\langle y_{5}\right\rangle$, and Rows B. 3 and B.9 of Table 2 combined together imply that $z_{2}=-y_{2}-x=-y_{6} \in\left\langle y_{5}\right\rangle$. Thus, since, by Row A.2 of Table 2, we have $y_{1}^{\alpha^{*}}=-y_{6}$, we can conclude that $\mathbb{Z}_{r}=\left\langle y_{5}\right\rangle$.

By Proposition 2.2 we can, without loss of generality, assume that $y_{5}=1$. Since, by Row B.9 of Table $2, z_{2}=-y_{2}-x$, the automorphism $\alpha^{*}$ gives that $y_{1}+z_{3}-y_{4}=y_{5}+x$ (see Rows A.1, A.3 and A.9 of Table 2) and so, since $y_{5}=-y_{4}$, we have that $x=y_{1}+z_{3}=$ $y_{5}+y_{6}$. Since $-x=x^{\alpha^{*}}=\left(y_{5}+y_{6}\right)^{\alpha^{*}}=-y_{1}-y_{2}$, it follows that $y_{1}+z_{3}=x=y_{1}+y_{2}$, and thus $z_{3}=y_{2}=z_{1}$. Row B.10 of Table 2 now implies that $0=y_{3}+y_{5}-y_{2}$. Since $y_{3}=-y_{2}$ it follows that $2 y_{2}=y_{5}=-y_{4}$. Applying Rows A.3 and A.5 of Table 2 to this equality gives that $-2 y_{5}=y_{3}=-y_{2}$. Therefore $y_{2}=2$, and consequently, by Row A.6 of Table 2, it follows that $1^{\alpha^{*}}=-2$. Now we get from Row A.1 of Table 2 that $-2 x=-x$, and so $x=0$. This implies that $y_{6}=-y_{5}=-1$ (since $\left.x=y_{5}+y_{6}\right)$. However, by Row B. 3 of Table $2, y_{6}=y_{2}+x=y_{2}=2$ and so $2=-1$. This shows that $r=3$, and therefore $X$ is isomorphic to F054A, the unique cubic symmetric graph of order 54 .

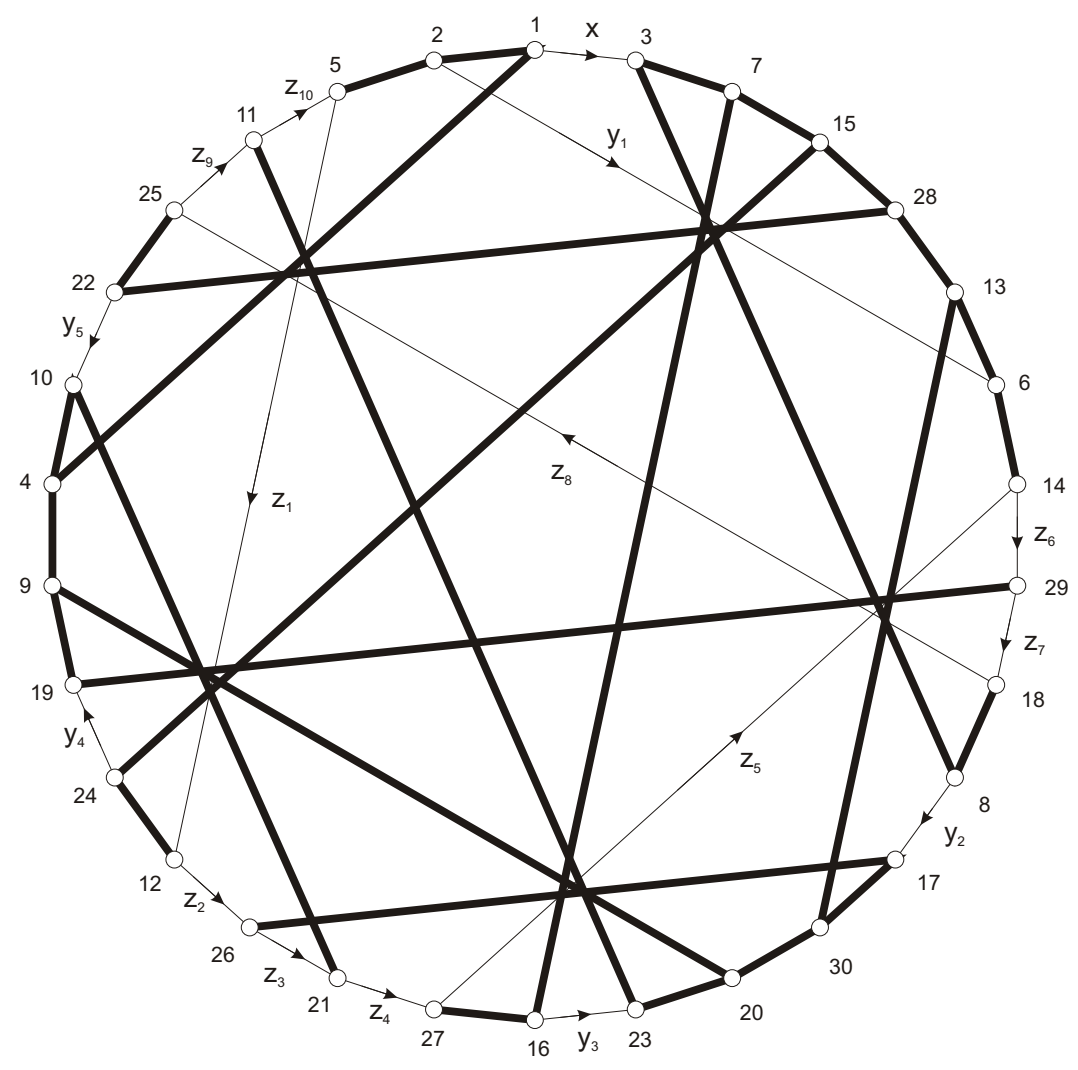

Figure 4: The voltage assignment $\zeta$ on F030A. The spanning tree consists of undirected bold edges, all carrying trivial voltage. 


\section{CASE $3 . X_{N} \cong \mathrm{F} 030 \mathrm{~A}$.}

The graph F030A is illustrated in Figure 4. It is known that F030A is the unique connected cubic symmetric graph of order 30 and that this graph is in fact 5-regular (see [4]). Each subgroup of AutF030A generated by a $(3,10)$-semiregular automorphism is conjugate to $\langle\beta\rangle$ where

$$
\begin{aligned}
\beta= & (1,3,7,15,28,13,30,20,9,4)(2,8,16,24,22,6,17,23,19,10) \\
& (5,18,27,12,25,14,26,11,29,21),
\end{aligned}
$$

and AutF030A has two proper arc-transitive subgroups, both of order 720 acting 4regularly. In addition, one of these two proper arc-transitive subgroups does not contain a $(3,10)$-semiregular automorphism whereas the other is generated by $\beta$ and

$$
\begin{aligned}
\alpha= & (2)(5)(11)(12)(23)(25)(1,6)(3,13)(4,14)(7,30)(8,28) \\
& (9,27)(10,29)(15,17)(16,20)(18,22)(19,21)(24,26) .
\end{aligned}
$$

These can be checked directly using Magma [2].

Let $H=\langle\alpha, \beta\rangle$. Then, in order to show that this case is impossible, that is, that $X$ is not a $\mathbb{Z}_{r}$-cover of F030A, it suffices to show that there is no connected $\mathbb{Z}_{r}$-cover $X$ of $F 030 A$ such that $\rho$ projects to $\beta$ (therefore, the lifts of $\beta$ centralizes the group $N$ of covering transformations) and that $H$ lifts to a subgroup of Aut $X$.

For this purpose observe that any such cover can be derived from F030A through a suitable voltage assignment $\zeta: A(\mathrm{~F} 030 \mathrm{~A}) \rightarrow \mathbb{Z}_{r}$. To find this voltage assignment $\zeta$ fix the spanning tree $T$ of $\mathrm{F} 030 \mathrm{~A}$ as the one consisting of the edges

$$
\begin{gathered}
\{1,2\},\{2,5\},\{3,8\},\{8,18\},\{7,16\},\{16,27\},\{15,24\},\{12,24\},\{22,28\},\{22,25\}, \\
\{6,13\},\{6,14\},\{17,30\},\{17,26\},\{20,23\},\{11,23\},\{9,19\},\{19,29\},\{4,10\},\{10,21\}, \\
\{3,7\},\{7,15\},\{15,28\},\{13,28\},\{13,30\},\{20,30\},\{9,20\},\{4,9\},\{1,4\} .
\end{gathered}
$$

(see also Figure 4). There are sixteen fundamental cycles in F030A, which are generated, respectively, by sixteen cotree arcs $(1,3),(2,6),(8,17),(16,23),(24,19),(22,10),(5,12)$, $(12,26),(26,21),(21,27),(27,14),(14,29),(29,18),(18,25),(25,11)$ and $(11,5)$ (see Table 3 where all these cycles and their voltages are listed).

The set $S=\left\{x, y_{i}, z_{j} \mid i \in\{1,2, \ldots, 5\}, j \in\{1,2, \ldots, 10\}\right\}$ of voltages of the sixteen fundamental cycles of F030A generates the voltage group $\mathbb{Z}_{r}$. By Proposition 2.3, the mapping $\bar{\beta}$ extends to the identity automorphism of $\mathbb{Z}_{r}$. Thus, Rows B.2 - B.6 of Table 3 imply that $y_{2}=y_{3}=y_{4}=y_{5}=-y_{1}$ and $x=2 y_{1}$, whereas Rows B.7 - B.16 of Table 3 imply that $z_{1}=z_{4}=z_{7}$ and $z_{2}=z_{3}=z_{5}=z_{6}=z_{8}=z_{9}=z_{10}=z_{1}-x$. It follows that $\mathbb{Z}_{r}=\left\langle y_{1}, z_{1}\right\rangle$. Moreover, applying the automorphism $\alpha^{*}$ to $z_{2}=z_{5}$ we get that $-z_{2}+y_{2}=x+y_{2}$ and so $x=-z_{2}$ (see Rows A.8 and A.11 of Table 3). This shows that $z_{1}=0$ and therefore $\mathbb{Z}_{r}=\left\langle y_{1}\right\rangle$. But, however, Row A.4 of Table 3 implies that $y_{3}^{\alpha^{*}}=0$, and so $y_{1}=-y_{3}=0$, a contradiction. 


\begin{tabular}{|c|c|c|c|c|c|}
\hline Row & & $C$ & $\zeta(C)$ & $C^{\alpha}$ & $\zeta\left(C^{\alpha}\right)$ \\
\hline A.1 & $C_{0}$ & $1,3,7,15,28,13,30,20,9,4,1$ & $x$ & $6,13,30,17,8,3,7,16,27,14,6$ & $-y_{2}+z_{5}$ \\
\hline A. 2 & $C_{1}$ & $1,2,6,13,30,20,9,4,1$ & $y_{1}$ & $6,2,1,3,7,16,27,14,6$ & $-y_{1}+x+z_{5}$ \\
\hline A.3 & $C_{2}$ & $3,8,17,30,13,28,15,7,3$ & $y_{2}$ & $13,28,15,7,3,8,17,30,13$ & $y_{2}$ \\
\hline A. 4 & $C_{3}$ & $7,16,23,20,30,13,28,15,7$ & $y_{3}$ & $30,20,23,16,7,3,8,17,30$ & $-y_{3}+y_{2}$ \\
\hline A.5 & $C_{4}$ & $15,24,19,9,20,30,13,28,15$ & $y_{4}$ & $17,26,21,27,16,7,3,8,17$ & $z_{3}+z_{4}+y_{2}$ \\
\hline A. 6 & $C_{5}$ & $28,22,10,4,9,20,30,13,28$ & $y_{5}$ & $8,18,29,14,27,16,7,3,8$ & $-z_{7}-z_{6}-z_{5}$ \\
\hline A. 7 & $C_{6}$ & $1,2,5,12,24,15,28,13,30,20,9,4,1$ & $z_{1}$ & $6,2,5,12,26,17,8,3,7,16,27,14,6$ & $-y_{1}+z_{1}+z_{2}-y_{2}+z_{5}$ \\
\hline A. 8 & $C_{7}$ & $15,24,12,26,17,30,13,28,15$ & $z_{2}$ & $17,26,12,24,15,7,3,8,17$ & $-z_{2}+y_{2}$ \\
\hline A.9 & $C_{8}$ & $30,17,26,21,10,4,9,20,30$ & $z_{3}$ & $7,15,24,19,29,14,27,16,7$ & $y_{4}-z_{6}-z_{5}$ \\
\hline A. 10 & $C_{9}$ & $4,10,21,27,16,7,15,28,13,30,20,9,4$ & $z_{4}$ & $14,29,19,9,20,30,17,8,3,7,16,27,14$ & $z_{6}-y_{2}+z_{5}$ \\
\hline A.11 & $C_{10}$ & $7,16,27,14,6,13,28,15,7$ & $z_{5}$ & $30,20,9,4,1,3,8,17,30$ & $x+y_{2}$ \\
\hline A. 12 & $C_{11}$ & $13,6,14,29,19,9,20,30,13$ & $z_{6}$ & $3,1,4,10,21,27,16,7,3$ & $-x+z_{4}$ \\
\hline A. 13 & $C_{12}$ & $9,19,29,18,8,3,7,15,28,13,30,20,9$ & $z_{7}$ & $27,21,10,22,28,13,30,17,8,3,7,16,27$ & $-z_{4}-y_{5}-y_{2}$ \\
\hline A. 14 & $C_{13}$ & $3,8,18,25,22,28,15,7,3$ & $z_{8}$ & $13,28,22,25,18,8,17,30,13$ & $-z_{8}+y_{2}$ \\
\hline A. 15 & $C_{14}$ & $28,22,25,11,23,20,30,13,28$ & $z_{9}$ & $8,18,25,11,23,16,7,3,8$ & $z_{8}+z_{9}-y_{3}$ \\
\hline \multirow[t]{2}{*}{ A.16 } & $C_{15}$ & $20,23,11,5,2,1,4,9,20$ & $z_{10}$ & $16,23,11,5,2,6,14,27,16$ & $y_{3}+z_{10}+y_{1}-z_{5}$ \\
\hline & & $C$ & $\zeta(C)$ & $C^{\beta}$ & $\zeta\left(C^{\beta}\right)$ \\
\hline B.1 & $C_{0}$ & $1,3,7,15,28,13,30,20,9,4,1$ & $x$ & $3,7,15,28,13,30,20,9,4,1,3$ & $x$ \\
\hline B.2 & $C_{1}$ & $1,2,6,13,30,20,9,4,1$ & $y_{1}$ & $3,8,17,30,20,9,4,1,3$ & $y_{2}+x$ \\
\hline B.3 & $C_{2}$ & $3,8,17,30,13,28,15,7,3$ & $y_{2}$ & $7,16,23,20,30,13,28,15,7$ & $y_{3}$ \\
\hline B. 4 & $C_{3}$ & $7,16,23,20,30,13,28,15,7$ & $y_{3}$ & $15,24,19,9,20,30,13,28,15$ & $y_{4}$ \\
\hline B.5 & $C_{4}$ & $15,24,19,9,20,30,13,28,15$ & $y_{4}$ & $28,22,10,4,9,20,30,13,28$ & $y_{5}$ \\
\hline B. 6 & $C_{5}$ & $28,22,10,4,9,20,30,13,28$ & $y_{5}$ & $13,6,2,1,4,9,20,30,13$ & $-y_{1}$ \\
\hline B.7 & $C_{6}$ & $1,2,5,12,24,15,28,13,30,20,9,4,1$ & $z_{1}$ & $3,8,18,25,22,28,13,30,20,9,4,1,3$ & $z_{8}+x$ \\
\hline B. 8 & $C_{7}$ & $15,24,12,26,17,30,13,28,15$ & $z_{2}$ & $28,22,25,11,23,20,30,13,28$ & $z_{9}$ \\
\hline B.9 & $C_{8}$ & $30,17,26,21,10,4,9,20,30$ & $z_{3}$ & $20,23,11,5,2,1,4,9,20$ & $z_{10}$ \\
\hline B. 10 & $C_{9}$ & $4,10,21,27,16,7,15,28,13,30,20,9,4$ & $z_{4}$ & $1,2,5,12,24,15,28,13,30,20,9,4,1$ & $z_{1}$ \\
\hline B.11 & $C_{10}$ & $7,16,27,14,6,13,28,15,7$ & $z_{5}$ & $15,24,12,26,17,30,13,28,15$ & $z_{2}$ \\
\hline B.12 & $C_{11}$ & $13,6,14,29,19,9,20,30,13$ & $z_{6}$ & $30,17,26,21,10,4,9,20,30$ & $z_{3}$ \\
\hline B.13 & $C_{12}$ & $9,19,29,18,8,3,7,15,28,13,30,20,9$ & $z_{7}$ & $4,10,21,27,16,7,15,28,13,30,20,9,4$ & $z_{4}$ \\
\hline B.14 & $C_{13}$ & $3,8,18,25,22,28,15,7,3$ & $z_{8}$ & $7,16,27,14,6,13,28,15,7$ & $z_{5}$ \\
\hline B.15 & $C_{14}$ & $28,22,25,11,23,20,30,13,28$ & $z_{9}$ & $13,6,14,29,19,9,20,30,13$ & $z_{6}$ \\
\hline B.16 & $C_{15}$ & $20,23,11,5,2,1,4,9,20$ & $z_{10}$ & $9,19,29,18,8,3,1,4,9$ & $z_{7}-x$ \\
\hline
\end{tabular}

Table 3: Fundamental cycles and their images with corresponding voltages in F030A. 


\section{References}

[1] N. Biggs, "Algebraic graph theory", 2nd ed., Cambridge University Press, Cambridge, 1993.

[2] W. Bosma, J. Cannon, and C. Playoust, The Magma Algebra System I: The User Language, J. Symbolic Comput. 24 (1997) 235-265.

[3] I. Z. Bouwer (ed.): The Foster Census, Winnipeg, 1988.

[4] M. D. E. Conder and P. Dobcsányi, Trivalent symmetric graphs on up to 768 vertices, J. Combin. Math. $\&$ Combin. Comp. 40 (2002), 41-63.

[5] S. F. Du, J. H. Kwak and M. Y. Xu, On 2-arc-transitive covers of complete graphs with covering transformation group $\mathbb{Z}_{p}^{3}$, J. Combin. Theory Ser. B 93 (2005), 73-93.

[6] R. Frucht, How to describe a graph, Ann. N. Y. Acad. Sci. 175 (1970), 159-167.

[7] R. Frucht, J. E. Graver and M. E. Watkins, The groups of the generalized Petersen graphs, Proc. Camb. Phil. Soc. 70 (1971), 211-218.

[8] H. H. Glover and D. Marušič, Hamiltonicity of cubic Cayley graph, J. Eur. Math. Soc. 9 (2007), $775-787$.

[9] J. L. Gross and T. W. Tucker, Generating all graph coverings by permutation voltage assignment, Discrete Math. 18 (1977), 273-283.

[10] J. L. Gross and T. W. Tucker, "Topological Graph Theory", Wiley - Interscience, New York, 1987.

[11] M. Herzog and G. Kaplan, Large cyclic subgroups contain non-trivial normal subgroups, J. Group Theory 4 (2001), 247-253.

[12] S. Hong, J. H. Kwak and J. Lee, Regular graph coverings whose covering transformation groups have the isomorphism extension property, Discrete Math 168 (1996), 85-105.

[13] R. Jajcay and J. Širán̆, Small vertex-transitive graphs of given degree and girth, Ars Math. Contemp. 4 (2011), 375-384.

[14] I. N. Kagno, Desargues' and Pappus' Graphs and Their Groups, Amer. J. Math. 69 (1947), 859-862.

[15] P. Lorimer, Vertex-transitive graphs: symmetric graphs of prime valency, J. Graph Theory 8 (1984), $55-68$.

[16] P. Lorimer, The construction of Tutte's 8-cage and the Conder graph, J. Graph Theory 13 (1989), $553-557$.

[17] A. Malnič, Group actions, coverings and lifts of automorphisms, Discrete Math. 182 (1998), 203218.

[18] A. Malnič, R. Nedela and M. Škoviera, Lifting graph automorphisms by voltage assignments, Europ. J. Combin. 21 (2000), 927-947.

[19] D. Marušič and T. Pisanski, Symmetries of hexagonal molecular graphs on the torus, Croat. Chem. Acta 73 (2000), 969-981.

[20] D. Marušič and T. Pisanski, The remarkable generalized Petersen graph G(8,3), Mathematica Slovaca 50 (2000), 117-121.

[21] T. Pisanski, A classification of cubic bicirculants, Discrete Math. 307 (2007), 567-578.

[22] W. T. Tutte, A family of cubical graphs, Proc. Cambridge Philos. Soc. 43 (1947), 459-474.

THE ELECTRONIC JOURNAL OF COMBINATORICS 19(2) (2012), \#P24 